\title{
Dynamics of Polarization Growth and Reversal in PVDF Films
}

\author{
M. Womes, E. Bihler \\ and W. Eisenmenger \\ Physikalisches Institut, Universität Stuttgart, \\ Stuttgart, Germany
}

\begin{abstract}
We present measurements of the time development of the dielectric displacement and the remanent polarization in PVDF for poling times ranging from $1 \mu \mathrm{s}$ to $1000 \mathrm{~s}$ and poling fields between 0.8 and $2.0 \mathrm{MV} / \mathrm{cm}$. For longer times $(0.1$ to $1000 \mathrm{~s})$ we determined also the time dependence of the polarization distribution across the film thickness. After applying a steep rectangular HV pulse, the sample is shorted to zero voltage. The remanent polarization under short-circuit conditions is compared with the maximum dielectric displacement under the external poling field. We observed a significant time delay of the build-up of the remanent polarization as compared to the dielectric displacement under field. This time delay depends significantly on the applied field strength and the crystallinity of the films. In the case of polarization reversal we observed for shorter poling times of up to $200 \mu \mathrm{s}$ a 'flipping back' of the polarization. Under these conditions, a large part of the polarization is reversed under the field, but after removal of the field, most of the polarization returns to the original direction. The results can be understood by the ferroelectric cooperative coupling of oriented crystallite dipoles to charges trapped at the surface of polarized crystallites. This, we think is responsible for the extremely high stability of the permanent polarization in PVDF.
\end{abstract}

\section{INTRODUCTION}

SINCE the discovery of the strong piezoelectric effect $S$ in PVDF by Kawai [1], significant progress has been achieved in understanding the basic mechanisms of the piezoelectricity (reviewed in $[2,3]$ ).

It is commonly accepted that the origin of the high piezoelectric coefficient in PVDF is attributed to the high polarity of the $\beta$-phase. The largest part of the piezoelectric response is due to a change in the density of the oriented crystallite dipole moments. The crystallite dipole moments can be permanently oriented by the application of high electric fields to PVDF-films. This and other properties indicate that PVDF is a 'hard' ferroelectric material. The cooperative dipole interaction within the crystallites primarily can explain 'soft' ferroelectricity. The hard ferroelectric properties are attributed to anisotropy fields of the near-hexagonal lattice of the $\beta$-crystallites [13]. This model predicts that the dipoles in crystallites switch between $60^{\circ}$ potential minima if an electric field is applied. Despite the success of this model some experimental observations are difficult to explain within its frame 
1. For lower poling fields strongly inhomogeneous spatial polarization distributions are found. The development of these polarization distributions observed during the poling process gives strong evidence that injection and migration of space charges determine the polarization distribution [12].

2. One of the most surprising features of the piezoelectricity in PVDF is its strong stability (i.e. the high coercive field strength) exceeding other ferroelectrics by almost three orders of magnitude [14].

3. A ferroelectric/paraelectric phase transition is not observed for PVDF. But the remanent polarization is lost upon heating the films above $80^{\circ} \mathrm{C}$.

Based on the work of Merz [6] on the switching in $\mathrm{BaTiO}_{3}$ single crystals, similar experiments were carried out with PVDF films to determine the switching times $[4,7]$.

As PVDF is regarded as a hard ferroelectric material with intrinsic potenţial barriers for dipole orientation it is not expected that the dielectric displacement and the accompanying remanent polarization show a different time dependence. Therefore in the switching experiments $[4,7]$ only the momentary electric displacement has been investigated, but not the remanent polarization as a function of different durations of the poling procedure. If instead the mechanism of the stabilization (i.e. making a change in polarization permanent) and of the momentary orientation of the crystallite dipoles are of different nature, we expect different time constants for both processes. We tried to answer this question by corresponding experiments. This is also of interest with respect to practical applications as, for example, ferroelectric data storage.

\section{EXPERIMENTAL}

$\mathrm{F}$ IGURE 1 shows the experimental arrangement. HV is applied to the sample by closing switch $\mathrm{S} 1$ and opening switch $\mathrm{S} 2$, shorting the sample is provided by the reverse operation. The switches $\mathrm{S} 1$ and $\mathrm{S} 2$ consist of field-effect power transistors and are triggered by external pulse generators.

The dielectric displacement $D$ at the end of the field pulse is calculated from the charge on capacitor $\mathrm{C},(D=$ $U_{C} C / A, A$ : sample area $=15.6 \mathrm{~mm}^{2}, C=10 \mathrm{nF}$ is large compared to the sample capacitance). The time dependence of the voltage $U_{C}$ at the capacitor $\mathrm{C}$ is shown in Figure 2 for a typical pulse experiment. The value

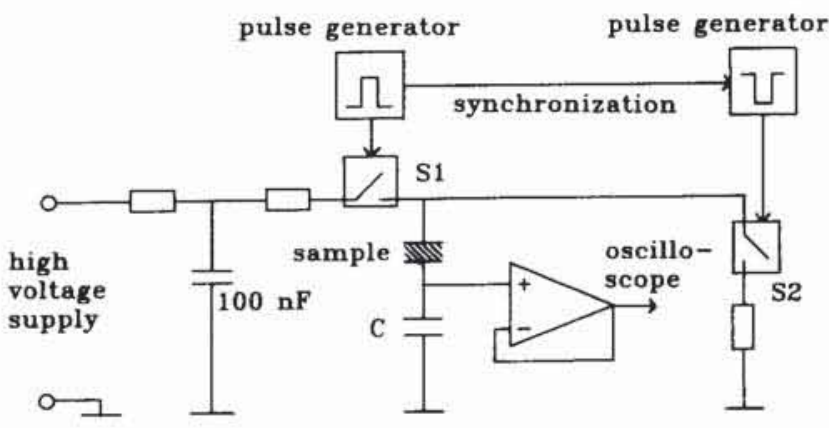

Figure 1.

Block diagram of the HV pulse generator used for poling the samples.

of the displacement $D$ is taken at the top of the final pulse edge. The remanent polarization $P$ caused by the pulse is measured after several minutes under short-circuit conditions with the PPS method (piezoelectrically generated pressure step) $[8,9]$. The result of this measurement is called the remanent polarization $P_{\text {rem }}$ in the further context. Of course the remanent polarization can be measured also via the voltage at capacitor C. Both methods have been compared carefully and agree within $10 \%$. We preferred the PPS-method for its convenience and for the possibility of observing the polarization distribution. For each of these measurements we used a new sample. Therefore in Figures 3-7 each data point corresponds to a different sample.

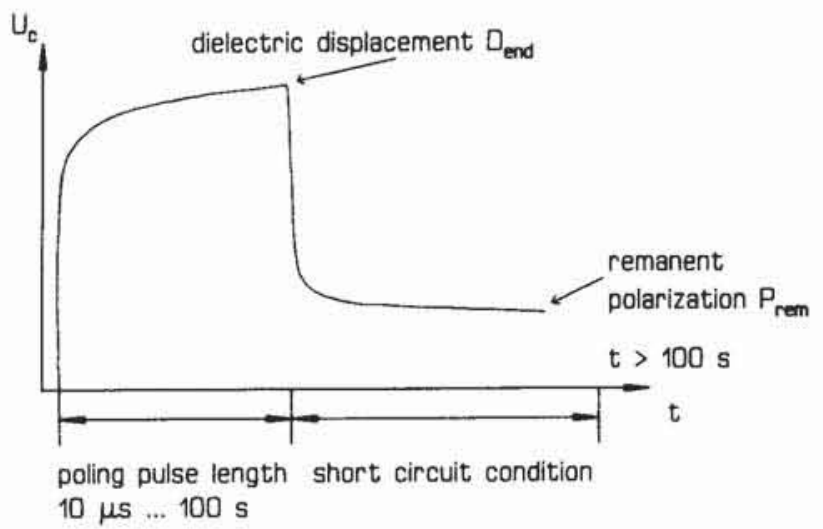

Figure 2.

Voltage at capacitor $\mathrm{C}$ for a typical pulse experiment.

The samples were $12 \mu \mathrm{m}$ thick PVDF films supplied from Kureha Chem., Japan (fabrication date 1975). This material contains $37 \%$ of its crystallites in the $\beta$-conformation. (The crystallite phase composition was obtained by IR-absorption measurement and evaluation according to Murayama [10]). Samples with a higher 
$\beta$ content of more than $85 \%$ were $9 \mu \mathrm{m}$ thick Sole ${ }^{\mathrm{m}}$ films supplied by Solvay, Belgium (1988).

\section{RESULTS}

\section{UNPOLARIZED FILMS}

$\mathrm{F}$ IGURE 3 shows the dielectric displacement $D$ and the remanent polarization $P_{\text {rem }}$ as a function of the pulse duration. The applied field strength is $2 \mathrm{MV} / \mathrm{cm}$. The development of the remanent polarization is significantly delayed against the fast build up of the dielectric displacement under the applied electric field. This is also evident by a comparison of the corresponding time constants. The displacement reaches half of its final value of $10 \mu \mathrm{C} / \mathrm{cm}^{2}$ after $200 \mu \mathrm{s}$, whereas the remanent polarization does not reach half of its final value of 4 $\mu \mathrm{C} / \mathrm{cm}^{2}$ before $100 \mathrm{~ms}$.

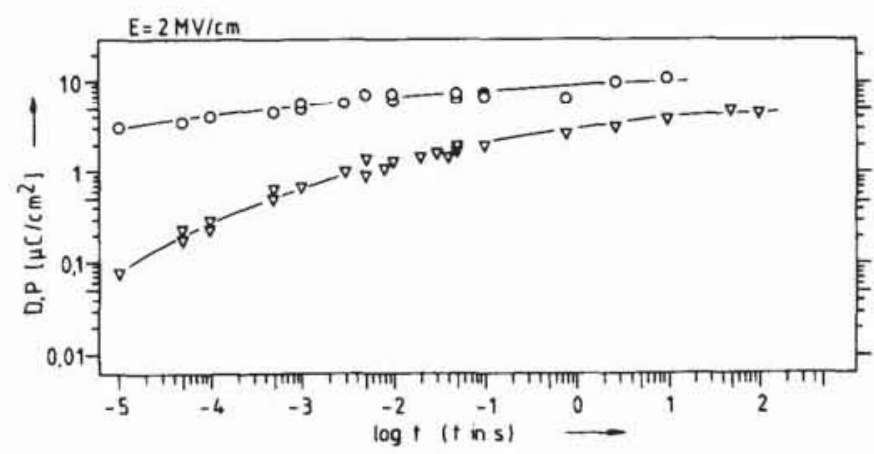

Figure 3.

Development of the displacement $D_{\text {end }}(000)$ and the remanent polarization $P_{\text {rem }}(\nabla \nabla \nabla)$, for unpolarized Kureha samples, $E=2 \mathrm{MV} / \mathrm{cm}$.

A comparison of Figure 3 with the results obtained under 1.2 MV $/ \mathrm{cm}$ (Figure 4) and $0.8 \mathrm{MV} / \mathrm{cm}$ (Figure 5) shows, that the time delay of the beginning development of the remanent polarization depends critically on the field strength. For a field strength of $2 \mathrm{MV} / \mathrm{cm}$ we observe a beginning remanent polarization above $30 \mathrm{nC} / \mathrm{cm}^{2}$ after poling with a $10 \mu$ s pulse, for $1.2 \mathrm{MV} / \mathrm{cm}$ after $10 \mathrm{~ms}$ and for $0.8 \mathrm{MV} / \mathrm{cm}$ after $1 \mathrm{~s}$.

\section{POLARIZED FILMS}

The poling procedure was the same as used by Furukawa and Johnson [4]. The samples were polarized

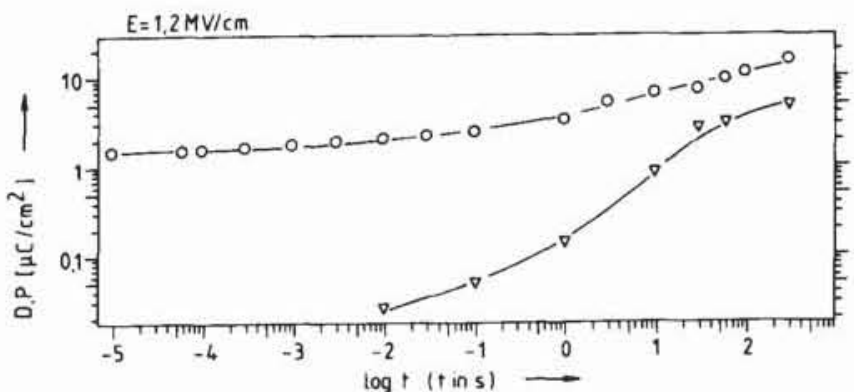

Figure 4.

Development of the displacement $D_{\text {end }}(\mathrm{OOO})$ and the remanent polarization $P_{\text {rem }}(\nabla \nabla \nabla)$, for unpolarized Kureha samples, $E=1.2 \mathrm{MV} / \mathrm{cm}$.

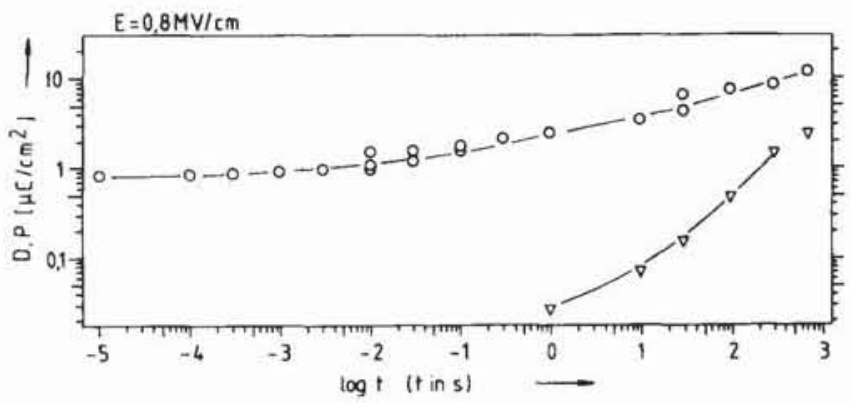

Figure 5.

Development of the displacement $D_{\text {end }}(\mathrm{OOO})$ and the remanent polarization $P_{\text {rem }}(\nabla \nabla \nabla)$, for unpolarized Kureha samples, $E=0.8 \mathrm{MV} / \mathrm{cm}$.

under $2 \mathrm{MV} / \mathrm{cm}$ for $100 \mathrm{~s}$ and then kept under shortcircuit condition for another $100 \mathrm{~s}$. After this procedure the samples had a remanent polarization of $4.8 \pm$ $10 \% \mu \mathrm{C} / \mathrm{cm}^{2}$. Then a reversely directed field pulse of $2 \mathrm{MV} / \mathrm{cm}$ was applied and the displacement $D$ and the remanent polarization $P_{\text {rem }}$ were measured as before. Figure 6 shows the result. After $200 \mu$ s the initial fast build-up of the displacement is finished and is continued by a slow ascent. Within this time interval the polarization under short-circuit conditions 'flips back' and the remanent polarization is still oriented in the original direction. The amount of remanent polarization is reduced with increasing pulse width, until after 200 $\mu$ s it starts growing in the new direction at the same time when the polarization under the external field saturates. This means that the orientation of dipoles and their stabilization are different processes, which occur on a different time scale. After having changed its sign, the remanent polarization grows faster and reaches a higher value after $100 \mathrm{~ms}$ as compared to the unpolarized samples (Figure 3). 


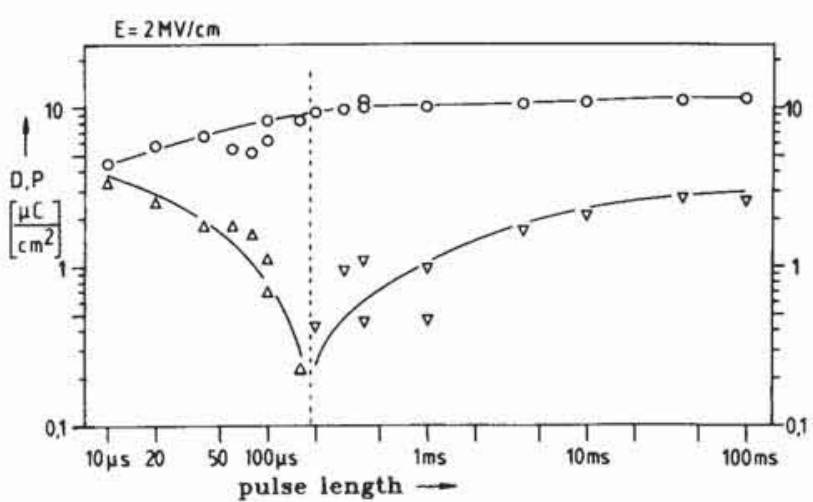

Figure 6 .

Reversal of the remanent polarization in prepolarized samples, Kureha $12 \mu \mathrm{m}$, applied field strength $2 \mathrm{MV} / \mathrm{cm}$. Displacement $D_{\text {end }}$ $(000)$, remanent polarization, original direction $(\Delta \Delta \Delta)$, remanent polarization, reversed direction $(\nabla \nabla \nabla)$. The dotted line indicates the time, when the remanent polarization crosses zero value.

Samples supplied from Solvay were prepared as the Kureha samples. The remanent polarization after the poling procedure was $6.8 \pm 10 \% \mu \mathrm{C} / \mathrm{cm}^{2}$. The results of the polarization reversal under an external field of $2 \mathrm{MV} / \mathrm{cm}$ are shown in Figure 7. The time delay of the remanent polarization is observed as with the Kureha samples, but the typical times are shorter. Zero remanent polarization has been reached after $10 \mu \mathrm{s}$. Also the increase of the displacement is faster.

Since at medium field strength $(1.2 \mathrm{MV} / \mathrm{cm})$ the poling process of the Kureha sample slowly takes place in seconds (see Figure 4), we were able to study the time development of the spatial polarization distribution using the PPS-response technique [9]. The HV was applied to the sample by a thin metalized PET-film. This allows the use of the PPS-response technique even during the poling process. The PPS-signals on the oscilloscope screen were recorded with a video system. Instead of using a new sample for each poling process with different pulse length the poling field now was applied in pulses of $100 \mathrm{~ms}$ duration and at a repetition rate of $1 \mathrm{~Hz}$. This is not completely equivalent to the poling by one continuous pulse. But it allows the analysis of the time-dependent development of the remanent polarization without changing the sample. During the time interval between subsequent pulses the sample was kept under short-circuit condition. The amplitudes of the displacement and the remanent polarization increase with

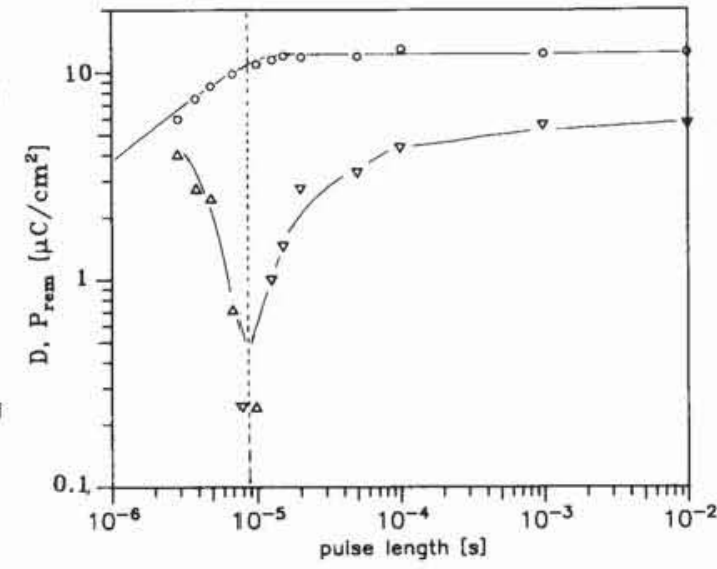

Figure 7.

Reversal of the remanent polarization in prepolarized samples, Solef $9 \mu \mathrm{m}$, applied field strength $2 \mathrm{MV} / \mathrm{cm}$. Displacement $D_{\text {end }}$ $(000)$, remanent polarization, original direction $(\Delta \Delta \Delta)$, remanent polarization, reversed direction $(\nabla \nabla \nabla)$. The dotted line indicates the time, when the remanent polarization crosses zero value.

increasing pulse number qualitatively similar as in Figure 3. The delayed development of the remanent polarization also was observed as before. Figure 8 shows the displacement distribution within the sample at the end of the 80 th pulse. The dotted line represents the corresponding remanent polarization after removal of the field. Apparently the polarization now is stabilized only in zones near the boundaries of the sample. The center of the sample is not permanently poled. This behavior is not observed for the $1.2 \mathrm{MV} / \mathrm{cm}$ continuous poling process discussed before, where we found a single, almost homogeneous zone. The same pulsed poling procedure was applied to prepolarized Kureha samples. Figure 9 shows the initial polarization distribution obtained after poling first for $100 \mathrm{~s}$ with a field strength of $2 \mathrm{MV} / \mathrm{cm}$. Within the spatial resolution of the PPS-apparatus (2 $\mu \mathrm{m})$, the sample is almost homogeneously poled. After the application of 80 pulses of reverse polarity (1.2 MV/ $\mathrm{cm}, 100 \mathrm{~ms}$ duration, $1 \mathrm{~Hz}$ frequency) the permanent polarization is reversed only in the center of the sample (dotted line in Figure 10). Near the boundaries the sample is still polarized in the original direction.

\section{DISCUSSION}

$\Gamma^{\text {HE }}$ significant delay of the development of the remanent polarization as compared to the fast build-up 


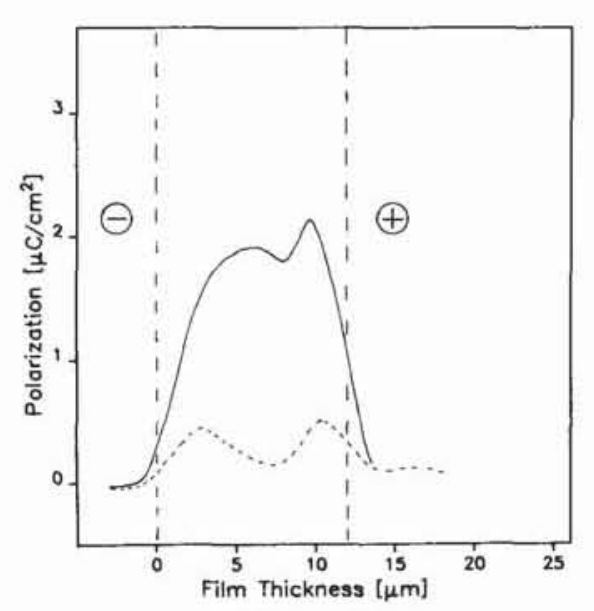

Figure 8.

Displacement (-) and remanent polarization (- . - ) after the application of 80 pulses. Field strength $1.2 \mathrm{MV} / \mathrm{cm}$, pulse duration $100 \mathrm{~ms}$, repetition rate $1 \mathrm{~Hz}$. The $+/-$ signs indicate the direction of the applied electric field.

of the displacement cannot be explained by a 6-site potential well model for dipole orientation [11] combined with cooperative behavior alone. In view of this model it would be expected that most of the dipoles stay in their new orientation after removing the poling field, independent of its duration. In contrast, our data show that in the case of unpolarized samples (Figure 3-5) the stabilization process only starts after a large amount of the crystallite dipoles have been oriented. The slow increase of the remanent polarization even in the time regime of seconds is presumably due to an electric fieldinduced phase transition of the $\alpha$ phase to the $\delta$ phase, since the Kureha samples have a low $\beta$ content of only $37 \%$.

For polarized samples (Figure 6,7) we find that the remanent polarization starts to grow in the new direction only after all dipoles are reversed under field.

The simplest model to explain the differing behavior of the orientation and stabilization process is to assume that the stabilization is not an intrinsic property of the dipole system but for example can be described by a model where the remanent polarization is fixed by trapped charges at the boundaries of the crystallites $[5,9]$. The number of deep traps in this case is assumed to be proportional to the polarization. The charges are trapped by dipole ends at the surfaces of the crystallites in PVDF (Figure 11). Within the frame of this model we understand that in unpolarized films the dipoles align under an external field, but rearrange after removal of the field if they are not stabilized by trapped charges. Since charges have to be injected into the polymer, the trapping is delayed against the development of the displacement (Figure 3). The dynamics of the dipole orientation under field as well as the injection current depend on the field strength. This leads to a strong field dependence of the growth of the remanent polarization (Figure 3-5). In prepolarized films the dipoles align fast under the external, reversing field with the new field direction. For short poling times the trapped charges cannot move very far from their sites, despite the fact that their trapping dipole-ends have disappeared. When the poling field is zero, these charges again orient the dipoles into their original direction being trapped again. For longer poling times the trap sites are destroyed more and more by the increasing dipole inversion and by increasing charge depletion at the original trapping sites. An alternative explanation for the displacement and remanent polarization development within the first 200 $\mu$ s (Figure 6) or $10 \mu$ s (Figure 7) is a model with two different dipole systems acting in parallel. One dipole system can be oriented fast. These dipoles may be attributed to crystallites which are not fixed in their orientation, or to amorphous dipolar chains. They have a random distribution of orientations. The dipoles, which form the remanent polarization, belong to the second dipole system. These dipoles cannot be oriented as fast as the first ones. They are oriented by their trapped charges. The displacement increase between 10 and 200 $\mu \mathrm{s}$ is attributed to the reorientation of these fixed dipoles, slowly loosing their trapped charges. As the displacement under the field increases, the 'flipping back' to the original remanent polarization under short-circuit conditions decreases. When the displacement saturates, all original trap sites are lost and the original remanent polarization has disappeared. To stabilize the growing new polarization with increased poling time, charges of opposite sign migrate and are trapped in new trap sites at the crystallite surfaces according to the opposite sign of the polarization. This leads to a slow and delayed reversal of the remanent polarization as compared to the faster build-up of the displacement (Figure 6,7). Since after destruction of the original permanent polarization the displacement has completely saturated, it is evident, that dipole orientation alone cannot be the reason for the stabilization of the polarization. The most simple mechanism already discussed and observed earlier $[5,9]$ is charge injection, charge transport and charge trapping at the surfaces of polarized crystallites. These charges also provide the necessary compensation charges for the zero-field condition. Therefore most charges are trapped at the boundaries of polarization zones. It is 
therefore expected that these boundaries are especially stable against polarization reversal.

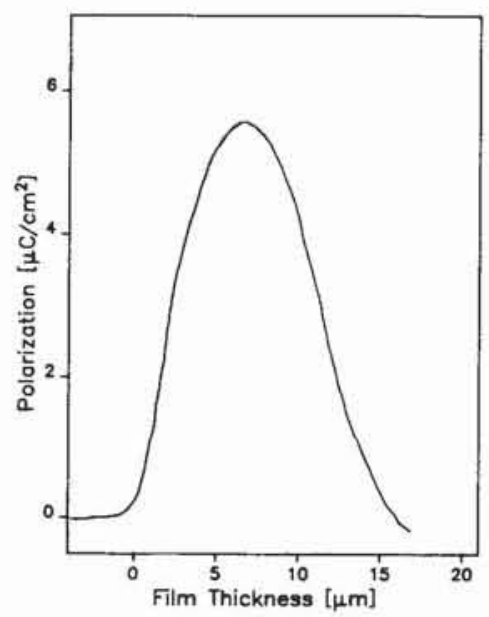

Figure 9.

Polarization distribution of a Kureha sample, thickness $12 \mu \mathrm{m}$, poled $100 \mathrm{~s}$ with $2 \mathrm{MV} / \mathrm{cm}$, before the polarization reversal (cp. Figure 10).

The rate of field-induced dipole chain rotation and the transport of the charges in the sample both depend on the field strength and consequently also the times necessary to reverse the remanent polarization. The faster development of the reversed remanent polarization in prepolarized films as compared to unpolarized films can be explained by the fact that injected charges are already present in prepolarized films.

The longer poling times necessary for permanent polarization of the Kureha material as compared to the Solvay films is attributed to the higher $\beta$ content of the latter. The probability for charge trapping is higher for material with large $\beta$ crystallite content. Therefore the charges have to migrate shorter distances into the polymer and the stabilization process is faster.

Additionally the electric field-induced phase transition from the $\alpha$ to the $\delta$ phase is important in samples with a smaller $\beta$ content. This process may lead to a slower increase of the displacement.

The polarization reversal with short pulses (Figure 10) shows that the polarization in the center of the sample can be reversed easier than that at the boundaries. This is consistent with our model, since charge trapping occurs primarily at the boundaries of the polarization zones. The dipoles which are adjacent to the trapped

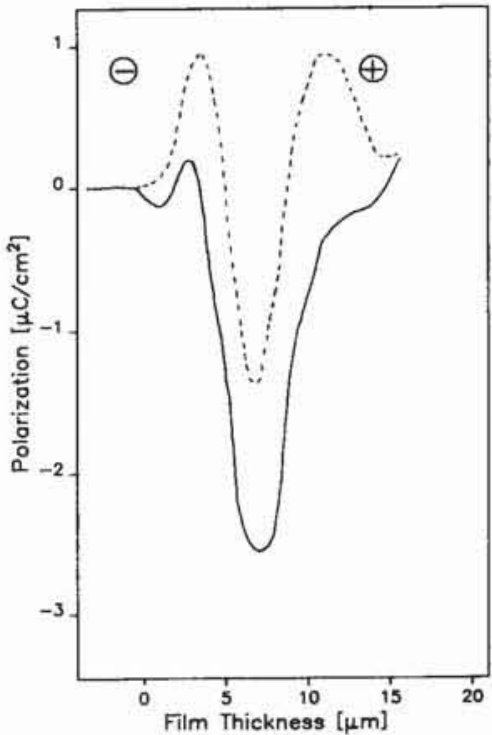

Figure 10.

Displacement (_ $)$ and remanent polarization (-.--) for a polarized sample (cp. Figure 9) after the application of 80 reverse field pulses. Field strength $1.2 \mathrm{MV} / \mathrm{cm}$, pulse duration $100 \mathrm{~ms}$, repetition rate $1 \mathrm{~Hz}$. The $+/-$ signs indicate the direction of the applied electric field.

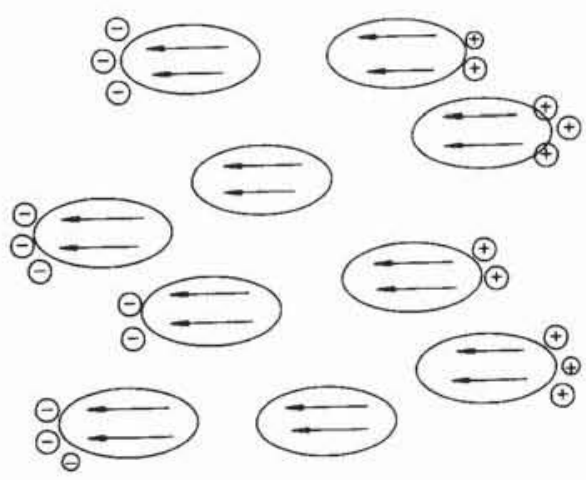

Figure 11.

Charge trapping model. The charges are trapped at the surface of crystallites at the boundaries of the polarization zone and vice versa stabilizing the oriented crystalline dipole moments.

charges are fixed stronger to their orientation. With medium fields not all of the traps can be emptied. The polarization zones at the boundaries therefore are more stable and keep their original direction. The dipoles in the center of the sample are first reoriented and then stabilized by newly trapped charges which are injected 
during the polarization reversal or released from former trap sites.

In discussing this interpretation of the result in Figure 10 it is important to exclude possible material inhomogeneities leading to a higher general stability of the polarization close to the film surface. This in fact is demonstrated by the result of Figure 8 , where an unpolarized sample of the same material polarizes first near the boundaries under otherwise identical conditions. This indicates that the dipoles in the boundaries of the sample in principle can be as easily oriented as in the rest of the sample. The reduced permanent polarization in the center of the sample in this case may be attributed to the possibility that in polarizing with multiple pulses, injected charges can migrate also to the film center during the zero-voltage time intervals. Under field pulse conditions these central charges reduce the central field strength and can be trapped also at the inside of the two polarization zones.

\section{CONCLUSION}

$\mathrm{T}$ HE observed experimental results cannot be explained alone by a cooperative or ferroelectric model with 6 site potential minima for the rotation of the chains in the polar crystallites. Instead it appears necessary that space charge injection and trapping have to be taken into account in order to arrive at a consistent description of the experiments. In our extended model, the oriented crystallite dipoles are fixed in their orientation by the field of trapped charges. The traps are associated with the dipole orientation since they are attributed to the dipole ends at the surfaces of polar crystallites. The strong stability of the polarization in PVDF appears as the result of the ferroelectric cooperative interaction between dipoles in the crystallites and trapped charges at the crystallite surfaces.

\section{ACKNOWLEDGEMENTS}

The authors are very grateful to K. Thonke, A. Dörnen and T. Wildermann for the IR measurements, to G. Eberle for the measurements on the Solef ${ }^{\mathrm{IM}}$ films and to D. K. Das-Gupta and G. M. Sessler for helpful discussions. Financial support of the Deutsche Forschungsgemeinschaft is gratefully acknowledged.

\section{REFERENCES}

[1] H. Kawai, "The Piezoelectricity of Poly(vinylidene Fluoride)", Japan J. Appl. Phys. Vol. 8, 975-976, 1969.

[2] R. G. Kepler, R. A. Anderson, "Piezoelectricity in Polymers", CRC Critical Reviews in Solid State and Materials Science, Vol. 9, 399-447, 1980.

[3] M. G. Broadhurst, G. T. Davis, "Piezo- and Pyroelectric Properties", Chapter 5 in: G. M. Sessler (ed.), Electrets, Springer Berlin 1980, 1987.

[4] T. Furukawa, G. E. Johnson, "Measurements of Ferroelectric Switching Characteristics in Polyvinylidene Fluoride", Appl. Phys. Lett., Vol. 38, 1027$1029,1981$.

[5] W. Eisenmenger, M. Haardt, K. Holdik, "Observation of Charge Compensated Polarization Zones in Polyvinylidenefluoride (PVDF) Piezoelectrically Generated Pressure Step Wave (PPS) Response", IEEE 1982 Annual Report Conf. Elect. Insul. and Dielect. Phen., pp. 52-57, Piscataway 1982.

[6] W. J. Merz, "Domain Formation and Domain Wall Motions in Ferroelectric $\mathrm{BaTiO}_{3}$ Single Crystals", Phys. Rev. Vol. 95, pp. 690-698, 1954.

[7] Y. Takase, A. Odajima, "Ferroelectric Polarization Switching in Polyvinylidene Fluoride", Japan J. Appl. Phys., Vol. 21, L707-L708, 1982.

[8] M. Haardt, W. Eisenmenger, "High Resolution Technique for Measuring Charge and Polarization Distributions in Dielectrics by Piezoelectrically Induced Pressure Step Waves (PPS)", IEEE 1982 Annual Report Conf. Elect. Insul. and Dielect. Phen., pp. 46-51, Piscataway 1982.

[9] W. Eisenmenger, M. Haardt, "Observation of Charge Compensated Polarization Zones in Polyvinylidenefluoride", Solid. State. Comm., Vol. 41, pp. 917-920, 1982.

[10] N. Murayama, "Persistent Polarization in Polyvinylidene Fluoride. I. Surface Charges and Piezoelectricity of Polyvinylidene Fluoride", J. Polymer Sci., Polymer Phys. Ed., Vol. 13, pp. 929-946, 1975.

[11] M. G. Broadhurst, G. T. Davis, "Ferroelectric Polarization in Polymers", Ferroelectrics, Vol. 32, pp. $177-180,1981$.

[12] K. Holdik, W. Eisenmenger, "Charge and Polarization Dynamics in Polymer Films", in G. M. Sessler 
(ed.), Proc. 5th Int. IEEE Symp. Electrets, Piscataway, pp. 553-558, 1985.

[13] R. G. Kepler, R. A. Anderson, "Ferroelectricity in Polyvinylidene Fluoride”, J. Appl. Phys., Vol. 49, pp. 1232-1235, 1978.

[14] G. M. Sessler, "Piezoelectricity in Polyvinylidene Fluoride", J. Acoust. Soc. Am., Vol. 70, pp. 1596$1608,1981$.
This paper is based on a presentation given at the 6th International Conference on Electrets, Oxford, England, 1-3 September 1988.

Manuscript was received on 30 Mar 1989 